\title{
Caring for Crying Babies: A mixed methods study to understand factors influencing nurses' and doctors' management of infant colic.
}

Original article

James McGann ${ }^{1}$ and Jaqueline Manohar1,2, Harriet Hiscock ${ }^{1,2,3}$, Denise O'Connor ${ }^{4}$, Jan Hodgson'1, Franz Babl1,2,3, Valerie Sung1,2,3

1Murdoch Childrens Research Institute, ${ }^{2}$ The Royal Children's Hospital, 3University of Melbourne Department of Paediatrics.

50 Flemington Road, Parkville, Victoria 3052

${ }^{4}$ Monash University, 553 St Kilda Rd, Melbourne, Victoria 3004

Corresponding author: Dr Valerie Sung

This is the author manuscript accepted for publication and has undergone full peer review but has not been through the copyediting, typesetting, pagination and proofreading process, which may lead to differences between this version and the Version of Record. Please cite this article as doi: $10.1111 /$ jpc.13858

This article is protected by copyright. All rights reserved. 
Royal Children's Hospital, 50 Flemington Road, Parkville, Victoria, 3052, Australia

Valerie.sung@rch.org.au

T: +61393454363

F: +6139345 5900

\section{Acknowledgments}

The authors would like to thank the participating Maternal Child Health Nurses and the Emergency Department staff members at The Royal Children's Hospital, Melbourne, Australia. Valerie Sung is supported by a National Health and Medical Research Council Early Career Fellowship (1125687) and The Royal Australasian College of Physicians Cottrell Research Establishment Fellowship, and was supported by a Melbourne Children's Campus Clinician Scientist Fellowship. Franz E. Babl's time was part funded by a grant from the Royal Children's Hospital Foundation, Melbourne, Australia. Harriet Hiscock is supported by an Australian National Health \& Medical Research Council (NHMRC) Career Development Award (Grant 607351). Infrastructure support was provided by the Victorian Government's Operational Infrastructure Support Program. 


\title{
$\underline{\text { Abstract }}$
}

\begin{abstract}
Aim: To determine the barriers and enablers influencing uptake of two recommendations from a tertiary paediatric hospital's clinical practice guidelines by Maternal Child Health Nurses (MCHNs) and Emergency Department (ED) doctors: a) explaining normal crying and b) avoiding attributing crying to gastroesophageal reflux (GOR) and limiting anti-reflux medication use.
\end{abstract}

Methods: One-hour focus group discussions, guided by the Theoretical Domains Framework, and a short questionnaire, with a purposive sample of MCHNs and ED doctors in Victoria, Australia in [months] 2015. Analyses by inductive content analysis to identify key barriers and enablers.

Results: 53 MCHNs and 25 ED doctors participated in 11 discussions. For explaining normal crying, key enablers were: adequate experience/competency, perceiving it was their role to explain and belief it prevented over-medicalisation. The main barriers were time restriction and beliefs about parents' perceptions. For MCHNs, key barriers to avoid attributing crying to GOR were: lack of knowledge and confusion around their role in diagnosing GOR. For ED doctors, key barriers to limiting anti-reflux medication were: parents requesting medication, concern about disrupting the parent-primary-care practitioner relationship and belief it was not their role to cease anti-reflux medication.

Conclusions: Overall, MCHN and ED doctors were proficient in describing normal crying. However, several barriers to best practice were identified, including time constraints and belief about consequences of intervening. These results will be used to develop effective interventions to address the identified barriers and enablers to optimise the management of infant colic. 


\section{What is already known on the topic}

- Infant colic is common, burdensome, causes significant distress to families and has no single medical intervention that is effective.

- Despite evidence that anti-reflux medications such as proton pump inhibitors are ineffective in reducing infant crying, they are widely prescribed for infant colic leading to unnecessary costs and potential harm to the infant.

- It is unclear what the barriers and enablers are for the successful implementation of existing clinical practice guidelines on how best to manage infant colic.

\section{What this paper adds}

- Key barriers of MCHNs and ED doctors to best practice management include time limitations, lack of understanding, reluctance to change care initiated by other clinicians: enablers include belief in avoiding over-medicalisation..

- Interventions that target these barriers and enablers could optimise patient care and enact change.

- This study highlights other areas that impact on how ED doctors and MCHNs manage infant colic, specifically parental perceptions and general practitioner prescribing practices, that require further investigation.

\section{$\underline{\text { Key words }}$}

Infant colic, excessive infant crying, Maternal Child Healthcare Nurse, Emergency

Department, Theoretical Domains Framework. 


\section{Introduction}

Infant colic, or excessive infant crying of unknown cause, is one of the most common reasons for parents to seek health professional help in the first three months of life.(1) Infant colic can be classified by Wessel's criteria, i.e.crying/fussing for at least 3 hours a day, for at least 3 days a week for at least 3 weeks.(2) The prevalence of cry-fuss problems is around 20\%(3) and it is a significant burden to the community. Excessive infant crying is the most proximal risk factor for the Shaken Baby Syndrome,(4) doubles to triples the risk of maternal and paternal depression $(5,6)$ and leads to the use of multiple healthcare services.(7) Despite extensive research, no single effective intervention exists for colic. Several clinical practice guidelines (CPGs) describe how to best manage excessive infant crying, such as the American Academy of Paediatrics guidelines(9), the United Kingdom (UK) NICE guidelines (10) and Australia's Royal Children's Hospital (RCH) guidelines from Melbourne.(11) All guidelines suggest excluding organic causes of crying and explaining normal infant crying patterns, whereby crying begins at around two weeks of age, peaks at around six to eight weeks of age and resolves between three to four months old.(12-14) The guidelines also recommend supporting the family and avoiding using non-evidence based medications, such as anti-reflux medications.

Despite the availability and dissemination of CPGs for managing infant colic, there is anecdotally wide variation in management practices between healthcare professionals. Child nurses have been found to offer inconsistent advice for infant crying often based on their personal opinions rather than professional judgement.(15) Health professionals, such as doctors and nurses, often label excessively crying infants with diagnoses, such as gastrooesophageal reflux (GOR).(16) However, there has been no proven causal link between GOR or gastro-oesophageal reflux disease (GORD) and crying in infants.(17) A systematic review on the effectiveness and safety of anti-reflux medications versus placebo in children with GORD showed no difference between groups in infant irritability or crying.(18) Despite the evidence, one study suggested $18 \%$ of Australian paediatricians report prescribing anti-reflux medications to infants for excessive crying.(19) A recent study that investigated Australian General Practitioners' (GPs') attitudes to GORD found that despite concerns regarding their safety and efficacy, GPs frequently prescribed anti-reflux medications.(20) While these medications are well tolerated short-term, there are suggestions of an association with increased susceptibility to infections and osteoporosis, and evidence about their long-term safety is lacking.(18) 
This wide variation in advice leads to unnecessary parental confusion and anxiety, adding to the burden of the condition. A unified approach from health professions to describing normal crying patterns to parents could reduce conflicting advice and subsequent parental confusion.(16) However, the publication of evidence-based CPGs alone does not appear to change management.(21)

Two healthcare workforces that play an important role in managing infant colic are Maternal and Child Health nurses (MCHNs) and Emergency Department (ED) doctors. MCHNs, qualified nurses and midwives with additional paediatric training, provide free healthcare to all children born in Victoria, Australia. The first MCHN scheduled visit is attended by $93 \%$ of all newborns.(22) Excessive crying is the second most common presentation (13.2\%) to Australian EDs for infants.(23) There are currently no studies exploring how MCHNs and ED doctors manage infant colic, whether they explain normal crying patterns or limit use of nonevidence-based medications. Further, no studies have investigated the barriers and enablers to evidence-based management of infant colic by MCHN and ED doctors.

Theory based interventions targeted to alter practice have been shown to affect change, as they focus on the most appropriate mediators of change that have been identified through investigation.(24) The Theoretical Domains Framework (TDF) is a method that provides a relatively easy yet comprehensive tool for identifying factors that influence healthcare professionals' behaviour.(25) Derived from 33 behavioural theories and 128 psychological constructs specific to healthcare, the TDF consists of 12 theoretical domains that can be explored when investigating implementation and behaviour change. The TDF is a validated method of examining healthcare professionals' behaviour(26) and has been used in various clinical areas.(27-34)

This study aimed to use a TDF guided approach to identify the barriers and enablers that influence the uptake by MCHNs and ED doctors of two key aspects of published infant colic CPGs: a) explanation of normal infant crying patterns and b) avoiding attributing GOR as a cause of infant crying or limiting the use of anti-reflux medications. This study also aimed to document these health professionals' awareness of the regional and national CPG from RCH, infant colic management practices and referral patterns.

\section{$\underline{\text { Materials and Methods }}$}

This article is protected by copyright. All rights reserved. 
This was a prospective mixed methods study. From March to September 2015, we conducted one-hour semi-structured focus group discussions, guided by the TDF, followed by a short questionnaire exploring participant demographics and infant colic management practices. We used purposive sampling to recruit nurses from MCHN centres and doctors from the RCH ED with all levels of training and years of experience. The RCH is a 385-bed tertiary care hospital and the ED sees 84,000 children annually. MCHNs were recruited from one of each of low, medium and high Socio-Economic Indexes for Areas (SEIFA) regions across the state of Victoria. SEIFA uses census data to rank areas in Australia according to relative socioeconomic advantage and disadvantage.(35)

The semi-structured discussion guide was developed using the TDF, with input from a behavioural change social scientist (D O'C), to explore factors influencing the two specific behaviours outlined in the CPGs. The script was piloted in a focus group discussion and refined following reflective analysis.

Participants were recruited via email invitation, facilitated by ED staff and MCHN regional managers. The two primary investigators (JMc, JMa) facilitated the focus group discussions with assistance from a supervisor (VS). Focus group discussions were audio-recorded, transcribed verbatim and validated by a member of the research team who deidentified participants and checked the transcripts against the original recordings for accuracy. The primary investigators coded the transcripts using a coding framework developed with input from experienced qualitative evidence-based researchers to ensure rigor and logical consistency.

1. We used inductive content analysis to generate specific coding factors (opinions, perspectives, actions, beliefs etc.) that impacted on desired behaviours.(36) We created a coding framework to group these specific factors spanning all focus groups. We then determined whether factors were 'barriers to change' or 'enablers of behaviour'.

2. We identified the importance of relevant factors within a specific behaviour by:

a. Frequency of mentions of that factor;

b. Importance placed on domain by participants, as perceived by the investigators; and

c. Clinical importance of domain as perceived by specialist paediatric researchers.

3. We mapped the relevant factors to TDF domains for each behaviour.

4. We listed the most important factors and their corresponding domains that impacted behaviour.

Any differences in interpretation were resolved through discussion. 
The study was approved by The RCH Human Research Ethics Committee (HREC 34272).

We reported the study according to the Standards of Reporting Qualitative Research.(37)

\section{$\underline{\text { Results }}$}

Seven consultants, four fellows (final year of specialty training) and 14 registrars (greater than 3 years of specialty training) participated in five focus groups for ED doctors. A total of $53 \mathrm{MCHNs}$ participated in six focus groups. Table 1 outlines the participant demographics.

\section{[Table 1]}

\section{Focus Group Discussions:}

\section{Explaining normal crying patterns: factors influencing practice.}

The vast majority of MCHN and ED doctors felt explaining normal crying was an important part of managing infant colic.

The key enablers that influenced explaining normal crying patterns were grouped into three main domains. Most participants felt confident and comfortable explaining normal crying (TDF Domain 'Beliefs about capabilities'). (Quotes \#1 and \#2)

Both MCHNs and ED doctors described a belief that explaining normal crying reassured parents ('Beliefs about consequences'). MCHNs felt it prevented over-medicalisation of the child (Quotes \#3 and \#4), and ED doctors believed it helped prevent representation to the ED (Quote \#6).

All MCHNs believed that it was their role to explain normal crying ('Role and identity'). When groups were asked directly if it was their role they replied "Definitely" and this was echoed across all groups (Quote \#5).

The major barrier to explaining normal crying was time restriction ('Environmental context and resources'). ED doctors and MCHNs overwhelmingly agreed that time limitation was one of the most difficult factors to navigate when managing crying infants (Quotes \#9, \#10 and $\# 11)$.

Another barrier identified by a few ED doctors was the parents' reluctance to listen to what normal crying was ('Beliefs about consequences'). They felt that by the time parents presented to the ED, they were not receptive to the explanation (Quote \#7). 


\section{[Table 2]}

The second CPG key behaviour is divided into two sections 1) avoiding attributing GOR as a cause of infant crying (MCHN specific) and 2) limiting the use of anti-reflux medication (ED doctors specific).

\section{Avoiding attributing GOR as a cause of infant crying: factors influencing practice (MCHN specific)}

The main enabler was minimising medicalisation of the child ('Beliefs about consequences'). MCHNs felt that normalising the crying and not attributing it to a specific diagnosis helped the family understand and manage the child. (Quote \#12).

The key barriers that influenced avoiding attributing GOR as a cause of infant crying were grouped into two main domains. Most MCHNs were unaware of the evidence that crying is not associated with GOR or GORD ('Knowledge'). Most MCHNs reported that GOR causes pain (Quote \#13).

Additionally, there was confusion around the MCHN's role in recognising and diagnosing GOR ('Role and identity'). The majority felt it was their role to recognise abnormal crying and refer on; however, confusion arose in where their role lay in discussing the symptoms and management of GOR and GORD with families (Quote \#16 and \#17).

\section{[Table 3]}

\section{Limiting the use of anti-reflux medication: factors influencing practice (ED doctors specific)}

The key enablers for ED doctors limiting the use of anti-reflux medications are grouped into three domains. First, the majority of participants were aware of both the ineffectiveness and the potential harms of anti-reflux medications ("Knowledge"; Quotes \#18 and \#19). Second, ED doctors, particularly senior clinicians, described regularly offering alternatives to medications as a normal part of their consultation process ("Behavioural regulation"; Quote \#20). Finally, participants felt that their colleagues within the ED were supportive of them avoiding anti-reflux medications ("Social influences"; Quote \#21).

When discussing barriers to limiting anti-reflux medications, it became clear that this key behaviour consisted of two distinct sub-behaviours for ED doctors; avoiding prescribing an anti-reflux medication in the ED and ceasing anti-reflux medication that had already been started. Participants described the pressures of prescribing a medication in ED due to parental 
desire for a prescription ("Social influences") and feeling the need to placate the parents by prescribing ("Beliefs about consequences"; Quotes \#22 and \#23). However, participants found it difficult to cease anti-reflux medications that the child was already on, often prescribed by GPs, prior to presenting to ED. While the majority of participants were aware that anti reflux agents are ineffective, they were hesitant to cease them as they believed that doing so would undermine the parent-GP relationship, which they perceived as more harmful to the child's overall health ("Beliefs about consequences"; Quote \#24). Several participants also expressed the view that it was not their role to stop these medications ("Role and identity"; Quote \#25).

\section{[Table 4].}

\section{Questionnaire:}

Table 5 outlines the MCHNs' and ED doctors' responses to the questionnaire. Most participating MCHNs (91\%) and ED doctors (96\%) were aware of The RCH CPG. However, guideline use varied. Almost $70 \%$ of ED doctors reported often or always explaining normal crying patterns and $76 \%$ reported their usual practice was never to prescribe anti-reflux medication. $69 \%$ of MCHNs reported suggesting anti-reflux medication sometimes while a small percentage (12\%) reported always suggesting them. Of note, $15 \%$ of MCHNs reported referring to chiropractors.

\section{[Table 5].}

\section{$\underline{\text { Discussion }}$}

This is the first study to examine the barriers and enablers of evidence-based management of infant colic by ED doctors and MCHNs. Both groups described similar enablers (adequate knowledge and finding it useful) and barriers (time restriction) to explaining normal crying. Several MCHNs were unaware of the evidence that GOR is not associated with infant crying. There was also confusion around their role in diagnosing GOR. Most ED doctors reported correct knowledge that GOR is not associated with crying, and reported processes to restrict the prescription of anti-reflux medications. However, several ED doctors reported reluctance in ceasing anti-reflux medications once they had been commenced. The questionnaire identified widespread knowledge of the regional and nationally used RCH CPG's existence but varied use by MCHNs and ED doctors.

This study has many strengths. It is the first study to examine infant colic management by Australian MCHNs and ED doctors. Our qualitative approach captured rich, novel data that 
have not previously been documented. We used a validated framework to systematically analyse the issue with representation from professions spanning several training levels and socio-economic locations. The RCH CPG is used nationally and is consistent with AAP and NICE guidelines.

This study also has some limitations. It relied on self-reported behaviours and participants' views or perceptions on what may influence practice. It potentially involves under-reporting of behaviours by participants in front of colleagues. We emphasised that all discussions were confidential and separated focus groups by seniority where possible to systematically examine behaviours and maximise honest contribution. Finally, the ED doctor component was only performed at a single-tertiary centre and thus may not be representative of other environments.

Whilst there is no literature with which to directly compare our results, findings are consistent with previous studies suggesting a wide variation in knowledge and management of infant colic by health professionals. $(15,38,39)$ The study identified that many MCHNs are unaware of the evidence that infant crying is not associated with GOR. This may lead to families being unnecessarily referred to multiple services and seeking non-evidence based, potentially harmful treatment for GOR. The study also indicated that $15 \%$ of MCHNs are recommending chiropractors for treatment of unsettled babies despite a systematic review finding no evidence to support its benefit in crying babies.(40)

ED doctors identified parental desire for prescription and disruption of the GP-parent relationship by ceasing a medication as key barriers affecting management. This cycle of misconception among parents as to the effectiveness of anti-reflux medications in colic and the over-prescribing by GPs highlights the need for further investigation into what may drive this belief within the community. This should include investigation into enablers and barriers of GP prescribing practices given a recent study suggesting that GPs are still frequently prescribing anti-reflux medications to crying infants.(20)

Universally, MCHN and ED doctors are proficient in describing normal crying patterns to families. However, this study identified that there is a wide variation across the groups in the management of infant colic. This study will inform future interventions to optimise the management of excessive infant crying by ED doctors and MCHNs. Successful intervention could limit unnecessary referrals for GOR/GORD, reduce conflicting advice and confusion for families, and ultimately reduce use of anti-reflux medications for managing infant crying. 


\section{References}

1. St James-Roberts I. Infant crying patterns in the first year: normal community and clinical findings. J Child Psychol Psychiatry. 1991;32(6):951-68.

2. Wessel MA CJ, Jackson ED, et al. Paroxysmal fussing in infancy, sometimes called colic. Pediatrics. 1954;14(5):421-35.

3. Wake M, Morton-Allen E, Poulakis Z, Hiscock H, Gallagher S, Oberklaid F.

Prevalence, stability, and outcomes of cry-fuss and sleep problems in the first 2 years of life: prospective community-based study. Pediatrics.117(3):836-42.

4. Reijneveld SA, van der Wal MF, Brugman E, Sing RAH, Verloove-Vanhorick SP. Infant crying and abuse. Lancet.364(9442):1340-2.

5. Smart J, Hiscock H. Early infant crying and sleeping problems: a pilot study of impact on parental well-being and parent-endorsed strategies for management. J Paediatr Child Health.43(4):284-90.

6. McMahon C, Barnett B, Kowalenko N, Tennant C, Don N. Postnatal depression, anxiety and unsettled infant behaviour. Australian \& New Zealand Journal of Psychiatry.35(5):581-8.

7. McCallum SM, Rowe HJ, Gurrin L, Quinlivan JA, Rosenthal DA, Fisher JR. Unsettled infant behaviour and health service use: a cross-sectional community survey in Melbourne, Australia. J Paediatr Child Health.47(11):818-23.

8. Ellis CL, Bokulich NA, Kalanetra KM, Mirmiran M, Elumalai J, Haapanen L, et al. Probiotic administration in congenital heart disease: a pilot study. J Perinatol.

2013;33(9):691-7.

9. Paediatrics AAo. Accessed online (11 July 2016) from http://pediatriccaresolutionsaaporg/contentaspx?resultClick=1\&gbosid=165497

10. NICE guidelines. Colic - infantile. Clinical Knowledge Summaries. Accessed online (8 January 2014) from http://cks.nice.org.uk/colic-infantile\#!management.

11. Guidelines R. Unsettled or crying babies (Colic). Accessed online (8 January 2015) from http://www.rch.org.au/clinicalguide/guideline_index/Crying_Baby_Infant_Distress/. 2012.

12. Lehtonen LA RP. Infantile colic: natural history and treatment. Curr Probl Pediatr. 1996;26:79.

13. TB B. Crying in infancy. Pediatrics. 1962;29:579-88.

14. RG B. The normal crying curve: what do we really know? Dev Med Child Neurol. 1990;32(4):356-62.

15. Hailes J LP, Wynter K, Rowe H, Burns J, Fisher J. Implementation of What Were We Thinking $(W W W T)$ in Victorian Maternal and Child Health services. Monash University, 2013. 
16. Douglas PS, Hiscock H. The unsettled baby: crying out for an integrated, multidisciplinary primary care approach. Med J Aust. 2010;193(9):533-6.

17. Wessel MA, Cobb JC, Jackson EB, Harris GS, Jr., Detwiler AC. Paroxysmal fussing in infancy, sometimes called colic. Pediatrics.14(5):421-35.

18. van der Pol RJ, Smits MJ, van Wijk MP, Omari TI, Tabbers MM, Benninga MA. Efficacy of proton-pump inhibitors in children with gastroesophageal reflux disease: a systematic review. Pediatrics. 2011;127(5):925-35.

19. Rimer R, Hiscock H. National survey of Australian paediatricians' approach to infant crying. J Paediatr Child Health. 2014;50(3):202-7.

20. Kirby C SA, Hinds R, Jones K, Piterman L. Infant gastro-oesophageal reflux disease (GORD): Australian GP attitudes and practices. J Paediatr Child Health. 2016;52:47-53.

21. Haines A DA. Making better use of research findings. BMJ. 1998;317:72-5.

22. Menchise AN, Cohen MB. Acid-reducing agents in infants and children: Friend or foe? JAMA Pediatrics. 2014.

23. Calado CS, Pereira AG, Santos VN, Castro MJ, Maio JF. What brings newborns to the emergency department?: a 1-year study. Pediatric Emergency Care.25(4):244-8.

24. Davies P WA, Grimshaw JM. A systemic review of the use of theory in the design of guideline dissemination and implementation strategies and interpretation of the results of rigorous evaluations. Implement Sci. 2010;10(5).

25. Michie S, Johnston M, Abraham C, Lawton R, Parker D, Walker A, et al. Making psychological theory useful for implementing evidence based practice: a consensus approach. Qual Saf Health Care. 2005;14(1):26-33.

26. Cane J, O'Connor D, Michie S. Validation of the theoretical domains framework for use in behaviour change and implementation research. Implementation Science. 2012;7:37. 27. Murphy K OCD, Browning C, et al. Understanding diagnosis and management of dementia and guideline implementation in general practice: a qualitative study using the theoretical domains framework. Implement Sci. 2014;9:31.(doi):10.1186/748-5908-9-31.

28. Bussieres AE, Patey AM, Francis JJ, Sales AE, Grimshaw JM, Canada PPT, et al. Identifying factors likely to influence compliance with diagnostic imaging guideline recommendations for spine disorders among chiropractors in North America: a focus group study using the Theoretical Domains Framework. Implementation Science.7:82.

29. French SD, McKenzie, Joanne E.; O'Connor, Denise A.; Grimshaw, Jeremy M; et al. Evaluation of a Theory-Informed Implementation Intervention for the Management of Acute Low Back Pain in General Medical Practice: The IMPLEMENT Cluster Randomised Trial. Plos-One. 2013;8(6).

30. Tavender E BM, Gruen R, Green S, et al. Understanding practice: the factors that influence management of mild traumatic brain injury in the emergency department-a 
qualitative study using the Theoretical Domains Framework. Implement Sci.

2014;9:8.(doi):10.1186/748-5908-9-8.

31. Boscart VM FG, Lee JH, Jaglal SB. Using psychological theory to inform methods to optimize the implementation of a hand hygiene intervention. Implement Sci.

2012;7:77.(doi):10.1186/748-5908-7-77.

32. Francis JJ, Stockton C, Eccles MP, Johnston M, Cuthbertson BH, Grimshaw JM, et al. Evidence-based selection of theories for designing behaviour change interventions: using methods based on theoretical construct domains to understand clinicians' blood transfusion behaviour. British Journal of Health Psychology.14(Pt 4):625-46.

33. Duncan EM, Francis JJ, Johnston M, Davey P, Maxwell S, McKay GA, et al. Learning curves, taking instructions, and patient safety: using a theoretical domains framework in an interview study to investigate prescribing errors among trainee doctors. Implementation Science.7:86.

34. Michie S, Pilling S, Garety P, Whitty P, Eccles MP, Johnston M, et al. Difficulties implementing a mental health guideline: an exploratory investigation using psychological theory. Implement Sci. 2007;2:8.

35. Statistics ABo. Socio-Economic Indexes for Areas 2013 [updated 23/7/2013; cited 2015 June]. Available from: http://www.abs.gov.au/websitedbs/censushome.nsf/home/seifa.

36. Elo S, Kyngas $\mathrm{H}$. The qualitative content analysis process. J Adv Nurs. 2008;62(1):107-15.

37. O'Brien BC, Harris IB, Beckman TJ, Reed DA, Cook DA. Standards for reporting qualitative research: a synthesis of recommendations. Academic Medicine: Journal Of The Association Of American Medical Colleges. 2014;89(9):1245-51.

38. Lobo ML, Kotzer AM, Keefe MR, Brady E, Deloian B, Froese-Fretz A, et al. Current beliefs and management strategies for treating infant colic. Journal of Pediatric Health Care. 2004;18(3):115-22.

39. Lobo ML, Kotzer AM, Keefe MR, Brady E, Deloian B, Froese-Fretz A, et al. Current beliefs and management strategies for treating infant colic. J Pediatr Health Care.

2004;18(3):115-22.

40. Ernst E. Chiropractic spinal manipulation for infant colic: a systematic review of randomised clinical trials. International Journal of Clinical Practice. 2009(9):1351. 


\section{University Library}

\section{- M M N E R VA A gateway to Melbourne's research publications}

Minerva Access is the Institutional Repository of The University of Melbourne

Author/s:

McGann, J;Manohar, J;Hiscock, H;O'Connor, D;Hodgson, J;Babl, F;Sung, V

Title:

Caring for crying babies: A mixed-methods study to understand factors influencing nurses' and doctors' management of infant colic

Date:

2018-06-01

Citation:

McGann, J., Manohar, J., Hiscock, H., O'Connor, D., Hodgson, J., Babl, F. \& Sung, V. (2018). Caring for crying babies: A mixed-methods study to understand factors influencing nurses' and doctors' management of infant colic. JOURNAL OF PAEDIATRICS AND CHILD HEALTH, 54 (6), pp.653-660. https://doi.org/10.1111/jpc.13858.

Persistent Link:

http://hdl.handle.net/11343/283643 